\title{
AUSTRALIA-JAPAN QZSS EMERGENCY WARNING SERVICE TRIAL PROJECT
}

\author{
S.Choy ${ }^{1, *}$, Y. B. Bai ${ }^{1}$, S. Zlatanova ${ }^{2}$, A. Diakite ${ }^{2}$, E. Rubinov ${ }^{3}$, C. Marshall ${ }^{3}$, P. Knight ${ }^{4}$, A. Riddell ${ }^{5}$, V. Rooke ${ }^{5}$, M. Woolf ${ }^{5}$, \\ N. Kishi ${ }^{6}$, S. Kogure ${ }^{6}$ \\ ${ }^{1}$ SPACE Research Centre, School of Science (Geospatial), RMIT University, Melbourne, Australia - (suelynn.choy, \\ yuntianbrian.bai)@rmit.edu.au \\ ${ }^{2}$ GRID, School of Built Environment, University of New South Wales, Sydney, Australia - (s.zlatanova, a.diakite)@unsw.edu.au \\ ${ }^{3}$ FrontierSI, Melbourne, Australia - (erubinov,cmarshall)@frontiersi.com.au \\ ${ }^{4}$ New South Wales Trade and Investment, Japan at Australian Trade and Investment Commission (Austrade), Sydney, Australia - \\ (peter.knight)@austrade.gov.au \\ ${ }^{5}$ Geoscience Australia, Canberra, Australia \\ ${ }^{6}$ QZSS Strategy Office, National Space Policy Secretariat, Cabinet Office, Government of Japan
}

\section{Commission IV}

KEY WORDS: Global and Regional Navigation Satellite Systems (GNSS/RNSS), Quasi Zenith Satellite System (QZSS), Emergency Warning Service (EWS), Emergency management

\begin{abstract}
:
This paper provides an overview and the results of the Australia-Japan 2020 Quasi Zenith Satellite System (QZSS) Emergency Warning System trial project. The project aimed to evaluate and demonstrate the feasibility of utilising the QZSS system to support emergency warning and response in Australia. The trial has focussed on bushfire and tsunami warnings with an emphasis on the message structure and standards for incorporation on the available signal bandwidth, and the spatial coverage extent of the messages. It also aimed to address the need for a space-based communication capability in Australia, which could potentially facilitate effective emergency warning system unconstrained by the limitations of terrestrial telecommunications.

A newly dedicated MobileApp was developed to decode the warning message and visualise relevant information on a map. Two messages for bushfire and tsunami warnings were generated in Australia and sent to the QZSS ground station for satellite transmission. The developed application was tested in Victoria and New South Wales. The trial was successful in the sense that the emergency warning message could be received and decoded using the QZSS enabled receivers and the dedicated MobileApp. The field tests showed that the systems are capable of delivering the required information to users with the required timeliness and completeness. Several technical issues encountered during testing can be primarily attributed to the alpha state of the app, and the specific receiver used for testing. Neither of which are considered to be significant barriers to the on-going development of an operational satellite EWS system.
\end{abstract}

\section{INTRODUCTION}

Australia is not immune to emergencies and disasters that are known to cause devastating human, economic and environmental losses. Having an emergency warning service in place allows the population to prepare, mitigate and enact emergency plans to respond and reduce the impact and loss experienced during an emergency or disaster. In Australia, the Australian National Emergency Alert, which is a national telephone-based service, enhanced with location-based capabilities, has been used widely since 2009. Nevertheless, telecommunications-based warning services are vulnerable to ground-based disruptions and network overload in the often extreme and unexpected circumstances surrounding an emergency and/or disaster. The current Australian emergency alert mechanism would benefit from an additional channel of delivery through space-based due to the large areas of the Australian landmass that lack mobile phone connectivity. Any space-based capability will not entirely replace the need for a telecommunications-based solution, however it will be able to augment the service and provide increased coverage.

\footnotetext{
${ }^{*}$ Corresponding author
}

Global and Regional Navigation Satellite Systems (GNSS/RNSS) and Satellite Based Augmentation Systems (SBAS), in addition to supporting Position, Navigation and Timing (PNT) applications, have the potential to delivery of short warning messages as part of the GNSS Emergency Warning Services (EWS). The Japanese Quasi Zenith Satellite System (QZSS) is an example of a RNSS developed to augment the United States Global Positioning System (GPS) for PNT applications and EWS in Japan, as well as the South East Asia and Oceania regions. As of September 2020, QZSS is a foursatellite augmentation system, three of which are in highly inclined elliptical orbits, and one in a geosynchronous orbit. When fully deployed in 2023, QZSS will consist of seven satellites providing 24-hour coverage to the region.

QZSS started providing emergency warning services in 2018 (Japan Cabinet Office, 2020). The service can handle two types of messages, one of which is the warning for large-scale events, coded according to an internationally agreed taxonomy of disaster types. Currently, operational services are available for the territory of Japan for tsunami. The message is composed by the Disaster Information Centre of the Japan Meteorological Agency and sent to the QZSS ground station, which transmits the message to the QZSS satellites. A receiver on the ground 
can receive the warning, decode and distribute it for visualisation to different users and the general public. The message is intended to be displayed either on signboards in public areas or on the car navigation dashboards for users registered for the service. The message can be also used to activate electric signboards along the seacoast.

In Australia there has been intermittent testing of GNSS satellite delivered EWS. In 2014-2015, a first project was undertaken with the aim to evaluate the potential utilisation of QZSS EWS for disaster alerting and warning purposes in Australia. Engagement was through primarily the Victorian state emergency agencies and Emergency Management Australia (Choy et al., 2016; Handmer et al., 2014). Then in 2018, Australia participated in the European Galileo-based Reliable Automatic and Low Latent Emergency (GRALLE) Warning Service project, which was tested with the Japanese QZSS system in Victoria, Australia over three days (GSA, 2018). The trial was successful, with messages able to be broadcast every four seconds, but begged questions about how the system could be operationalized in Australia.

In 2020, a follow on collaborative research partnership between the Australia and Japan was established to further build capacity in Australia and investigate the feasibility of using the QZSS EWS technology in the Australian emergency management community. The testing follows on from the 2019/2020 bushfire season, which has been widely documented as one of the worst on record. At the beginning of February 2020, over 11 million hectares of bush, parks and forest have been burnt with the unfortunate loss of 33 lives, and significant impact to native fauna.

This has prompted a renewed interest in a satellite-delivered EWS capabilities provided by QZSS. FrontierSI together with Geoscience Australia (GA), Australian Trade and Investment Commission (Austrade), the Japanese National Space Policy Secretariat (NSPS), RMIT and UNSW Universities, NSW State Emergency Services (SES), NSW Rural Fire Service (RFS) and Emergency Management Australia (EMA) have collaborated in Australia-Japan 2020 QZSS EWS trial project. This paper provides an overview of the trial project, as well as a summary of the results obtained, conclusions drawn, and recommendations made.

\section{GNSS EWS TECHNOLOGY}

GNSS EWS combines the strengths of both mobile telecommunication services and satellite-based communication to disseminate emergency warning messages. The GNSS receiver embedded in mobile devices such as smartphones providing information of the users' position could be used to correlate messages sent from the satellite based on the message geographical criteria (Choy et al., 2016). This makes the information relevant to intended users at a specific point in time and within the defined geographical area. GNSS could be used to supplement and enhance exiting warning systems. For example, Europe has been working on emergency message services since 2005 using the EGNOS and Galileo satellite navigation systems with the introduction of the ALIVE (Alert interface via EGNOS) Concept (Mathur et al., 2005). Since then there were follow on projects investigating technical and nontechnical benefits as well as advantages of utilising GNSS satellites for disaster alerting (Wallner, 2011; Domínguez et al., 2013).
A feature of the QZSS provision for alert messaging is that in addition to the wide area coverage provided by the satellite system, the receivers also provide, through their embedded GNSS/GPS capabilities, precise position information. In this way, alert messages can be sent to a specific area depending on the type and content of the disaster information, and only those receivers within the specific area will be activated (Iwaizumi, Kohtake, 2013; Iwaizumi et al., 2014). Knowing the area of the possible disaster location, the intended users could then be warned, while those outside the disaster area would not be alerted.

The satellite-based system offers a number of advantages for real-time disaster alerts over current approaches to sending warnings via personal devices. A disadvantage is that at present the signal is not well received indoors and urban canyons due to the low signal strength (GPS signals do not typically pass through solid objects). Advantages include:

(1) GNSS with location-based information can be used during an emergency. This provides spatial awareness whereby the messages can be applied to the EWS device's location;

(2) The service can cover a wide area simultaneously - e.g. the whole of Australia - because of its wide area broadcast footprint, and within the broadcast area, there is no limit to the number of people who can be warned simultaneously;

(3) The messages can still be received even when terrestrial communications infrastructure is damaged or not available. This allows for additional redundancy; and

(4) As the system is independent of mobile phone coverage it would reach people wherever they are, regardless of the existence of mobile phone coverage.

Figure 1 illustrates an example of a GNSS EWS system and basic process flow: First, an emergency or natural hazard occurs, e.g. bushfire, heavy rain/flood, strong weather, tsunami etc. Then, observations and data are collected by the relevant emergency service and management organisations about the emergency/hazard, e.g., about its location, intensity, type etc. This can be a combination of various data collection and observation techniques. The Emergency Service Centre validates and identifies a distribution area for the broadcast and provides details of the event to the Message Control Centre. The Message Control Centre compiles the message and transmits this to the satellite via the ground segment. The satellite then transmits the warning messages to all mobile phones within the satellite coverage area. The alert warning message is triggered on the end-user device if the end-user is within the target area.

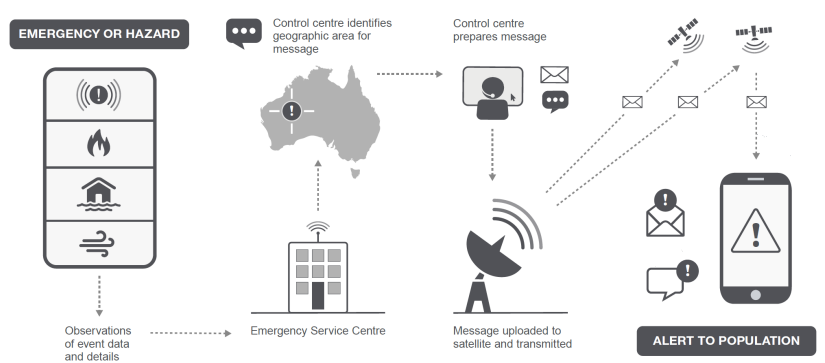

Figure 1. Basic concept of a GNSS EWS transmission system 


\section{AUSTRALIA-JAPAN QZSS EWS TRIAL PROJECT}

\subsection{QZSS EWS Enabled Equipment}

Three GNSS receivers were provided by the Japanese company Softbank to support the Australia-Japan QZSS EWS trial project. The receiver is a combined GPS and QZSS receiver, capable of providing position information and at the same time it is able to also receives and decodes the QZSS EWS messages. The Softbank receiver, shown in Figure 2 has an internal battery power source and can be connected to a mobile phone or PC via USB cable.

The Softbank receivers are quite simplistic and do not have any software associated with them as they simply output various message strings via the USB to a suitable serial terminal. In order to support field tests with emergency services personnel, it was necessary to develop a test interface mobile application (MobileApp) that could import the QZSS EWS messages from the Softbank receiver, $\log$ and decode them, and show the extents of decoded events on a map view. It was decided to build the MobileApp on the Android platform due to the wide range of Android devices being used, and the simplified development environment. In future, the MobileApp could be expanded to other platforms such as Windows and iOS.

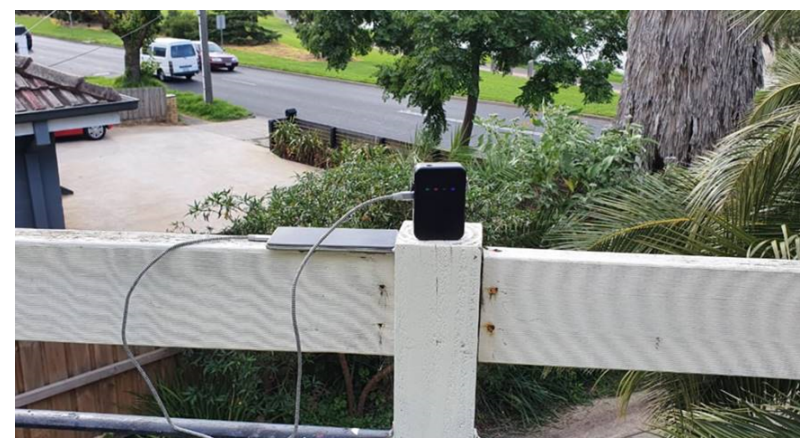

Figure 2. The Softbank GNSS QZSS enabled receiver used in the EWS trial project

\subsection{QZSS Signal Testing Methodology}

The QZSS EWS signal trial project comprised of a number of technical tests. Firstly, a bench test was completed to ensure a stable workflow from the design and transmission of Australian EWS messages via QZSS, to receiving, decoding and logging of the EWS message data.

Once the bench test was completed successfully, the research teams from RMIT and UNSW Universities developed an Android MobileApp, needed to extract and decode the EWS message contents based on the logged satellite messages.

After the MobileApp had been developed, members of SES and RFS carried out a field-testing campaign. A suitable area in NSW was chosen to allow overlap of emergency event regions, and test messages were modified to match the test area. A test campaign was designed, involving two alternating emergency events scheduled at specified times over two separate periods, focussing on metropolitan Melbourne and Sydney. Initial trials during the first test period indicated successful reception and decoding of the designed EWS messages, allowing progression to field trials with Emergency Services personnel.

During each test, NSPS successfully transmitted the preprogrammed test messages designed in Australia at the specified times. The SES and RFS members were equipped with the Softbank receivers and access to the MobileApp, and used them in the field to test the effectiveness of the system.

These steps are described in detail below:

\subsubsection{Bench Test}

The first phase of the project involved familiarisation with the Softbank receivers to understand their operational capabilities. After initial testing, a test EWS message was developed together with NSPS and was transmitted at an agreed time on $16^{\text {th }}$ July 2020 . The purpose of the initial test was to:

- test the basic capabilities of the Softbank receivers;

- ensure the receivers are able to track the EWS signal correctly;

- conduct a limited study of the geographic coverage in which messages could be received;

\subsubsection{Android MobileApp Development}

Based on the data logged by the Softbank receivers, the research team from RMIT and UNSW Universities developed an Android MobileApp to decode the emergency messages. The emergency messages contain standardised information of 21 items (Table 1) of which message type, countryID, providerID, event category, event sub-category, severity, start time of disaster (event onset day, hour, minute), duration, guidance response type, event location (latitude, longitude) and area of effect (semi-major, semi-minor, azimuth) were used in the pilot.

\begin{tabular}{|l|l|l|}
\hline Message & Binary Value & Description \\
\hline Message Type & 10 & Test \\
\hline Country ID & 0111110111 & Australia \\
\hline Provider ID & 0000 & RFS \\
\hline Event Category & 111 & Fire/Env/Infra \\
\hline Event Sub-Category & 000 & Forest Fire \\
\hline Severity & 11 & Minor \\
\hline Event Onset Day & 10000 & 16 \\
\hline Event Onset Hour & 00001 & 1 am UTC \\
\hline Event Onset Minute & 000000 & 0 \\
\hline Duration & 0111 & $2-3$ hours \\
\hline Guidance Library & 00 & International \\
\hline Response type & 0000 & Evacuate \\
\hline Instructions & 1000 & Spare \\
\hline Latitude & 010010100100011 & -37.775 \\
\hline Longitude & 1110011100010110 & 144.975 \\
\hline Semi-major & 0110 & $10000 \mathrm{~m}$ \\
\hline Semi-minor & 0110 & $10000 \mathrm{~m}$ \\
\hline Azimuth & 0000000 & 0 \\
\hline Specific Setting & 000000000000000000000 & To be left blank \\
\hline
\end{tabular}

Table 1. Emergency message structure

The user location was obtained as well from other available GNSS. The emergency message is decoded in 122 bits (out of 124) and transmitted as hexadecimal message. Examples of the tsunami and Bush fire messages are given below:

\section{- Tsunami 2239B41275C00E00610807DE \\ - Forest/Bushfire CDCE2C948E0070030C707DE}

The decoded information is continuously visualised in the MobileApp as a text message and displayed on a map interface for use in the field tests.

The MobileApp in its alpha stage basically has the following functionality: 
- import data from the Softbank (or any other compatible) receiver;

- decode and display the contents of the message;

- use geofencing to compare the user's position to check proximity to the event;

- if the user is within the radius of the event, the app displayed the event on the map; and

- $\log$ event information to a file for further analysis.

\subsubsection{Field Tests with Emergency Personnel}

After the initial test of the Softbank receiver equipment and the mobile app development, the alpha prototype system was ready to be trialled by the SES and RFS personnel. The purpose of the test was mainly to:

- confirm that the system consisting of both the Softbank receiver and the MobileApp worked in the field, and

- receive feedback on the practicality of the GNSS EWS signal and functionality of the MobileApp.

The field test took place from $12-13^{\text {th }}$ August 2020. On $12^{\text {th }}$ August, the UNSW team conducted the test and checked that the equipment and the newly developed MobileApp were working as expected. On $13^{\text {th }}$ August, members of the SES and RFS teams carried out the final testing across metropolitan Sydney.

Similarly to the Melbourne test, two events were created, i.e., one for tsunami and one bushfire. A map indicating the epicentre and extents of the emergency events is shown in Figure 3.

Both SES and RFS personnel were to move in and out of the designated emergency areas and observe the app behaviour during the test period to see if the app could correctly identify and display the nearby emergency events in real-time.

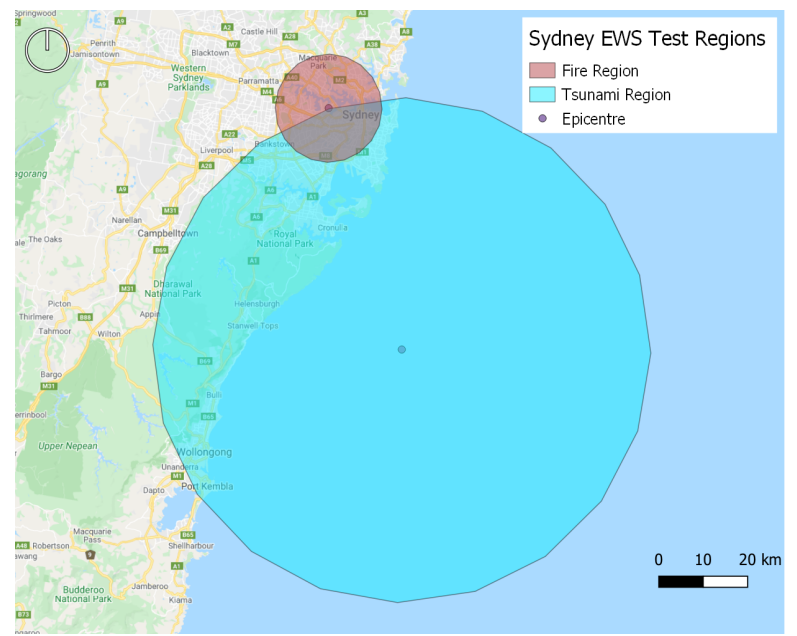

Figure 3. Map of the Sydney emergency events

\section{TEST RESULTS}

\subsection{Bench Test Results}

The initial test was carried out in Melbourne and Sydney on 16th July 2020. Two warning messages were created for the purpose of the test, one representing a tsunami, and the other representing a bushfire. A total of three sites were tested using two of the receivers in Melbourne - one within the vicinity of both events, one within the vicinity of bushfire event only, and the third site was out of the range of both events. Logging was also conducted in transit between the sites for later analysis. Additionally, another receiver was set up in Sydney to verify whether the messages would also be received in a geographic area a long distance from the emergency event. The test was carried out between $10 \mathrm{am}$ and $4 \mathrm{pm}$ AEST for a total period of six hours with the emergency message alternating each hour. The map in Figure 4 below shows the locations in which EWS messages were received in Melbourne.

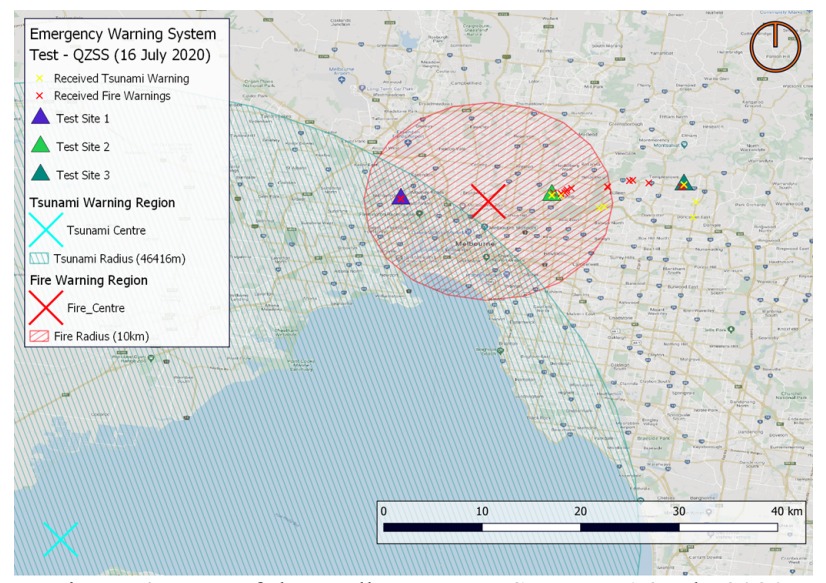

Figure 4. Map of the Melbourne EWS test on 16 July 2020

Results demonstrated that both messages were received successfully by all three receivers including the receiver in Sydney. This indicates that the satellite broadcasts the messages across the whole footprint area. Anyone within this footprint area will be able to receive them, whether they are close to the emergency area or not. That means the MobileApp is required to filter out the local messages based on the user's geographical location.

\subsection{EWS MobileApp}

After the completion of the initial test in Melbourne, the research teams at RMIT and UNSW universities worked on developing the MobileApp for the EWS testing with the capabilities described in Section 3.2.2. The data that was logged during the bench test was used for the app development process.

It should be noted that the teams had very limited window of time for development of approximately four to six weeks, so the main focus was on generating a working version of the MobileApp before the field-test with emergency personnel in Sydney. Consequently, the MobileApp was still very much in an alpha stage with only the basic functionality available. A number of deficiencies noted during testing were a result of the rudimentary MobileApp development, and the limited resource inputs to develop it. Some of the screenshots from the app are shown in Figure 5 and Figure 6 below. 


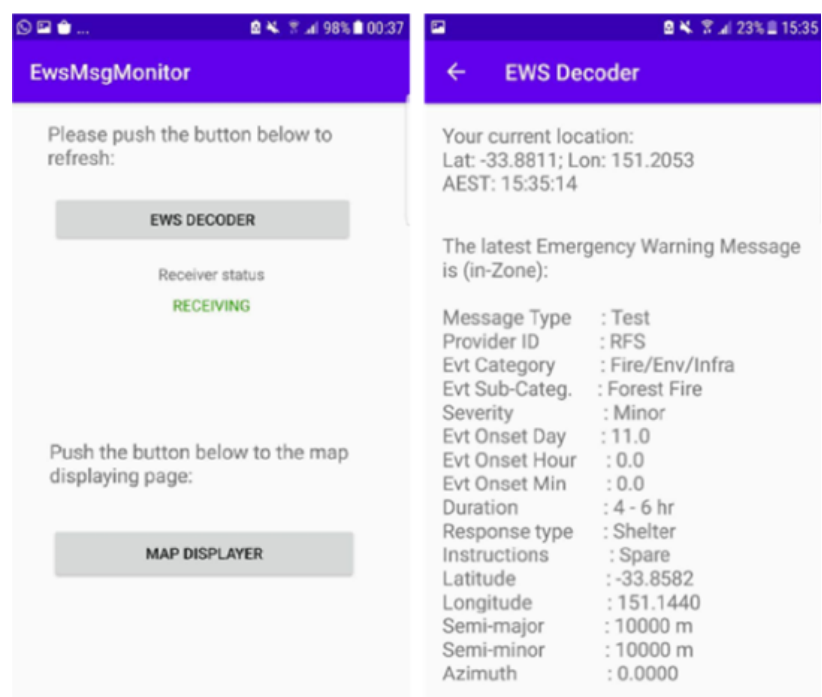

Figure 5. Screenshots from the EWS Android App

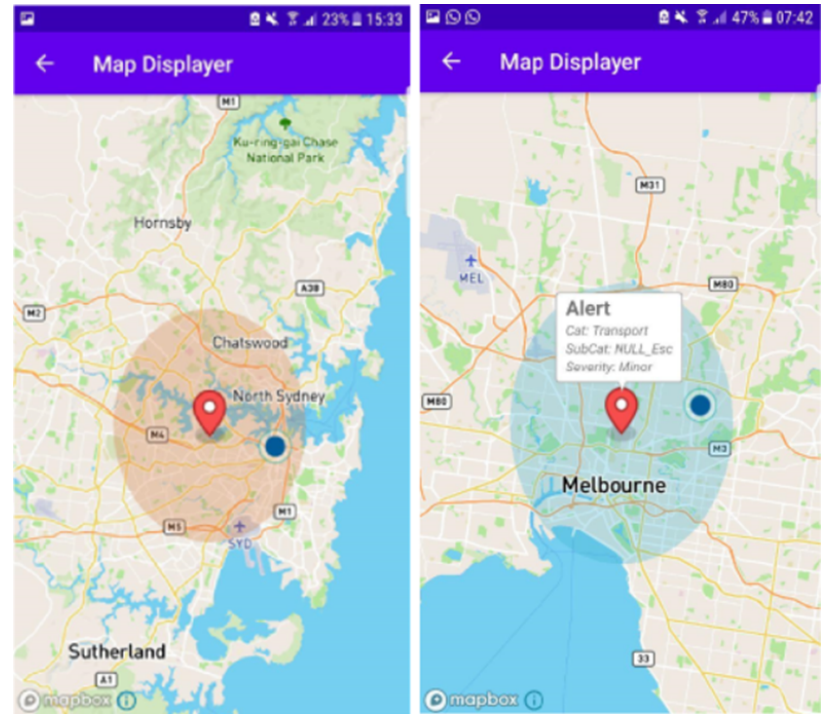

Figure 6. Screenshots from the EWS Android App

\subsection{EWS test with emergency personnel in Sydney}

Two emergency personnel, one from NSW SES and one from NSW RFS, were involved with conducting the EWS testing in Sydney on 13th August. The staffs were provided with a test methodology ahead of the tests, describing the test areas, and a schedule of the alternating emergency messages.

The three criteria that were being tested included:

- ability of the equipment to receive, decode and log QZSS EWS messages;

- ability of the MobileApp to identify user location relative to the event;

- ability of the MobileApp to display real-time alerts based on user's proximity to the event

All criteria were tested inside and outside the emergency areas. At the completion of the test, it was found that inside the emergency area the MobileApp was successful in achieving all three criteria; and outside of the emergency area some minor issues were encountered with identifying the user location relative to the event, but other than that, it could also be identified as successful. These minor issues were related to the alpha state of the MobileApp and are discussed in more details in the next section.

Figure 7 shows a portion of the route taken by the NSW SES personnel during the test, however, is not indicative of the period in which the EWS decoder app was logging. The yellow circles show where the emergency alert messages were successfully received and logged to disk on the tester's android phone. This includes both Tsunami and Fire messages, which are filtered for relevance at the app-level.

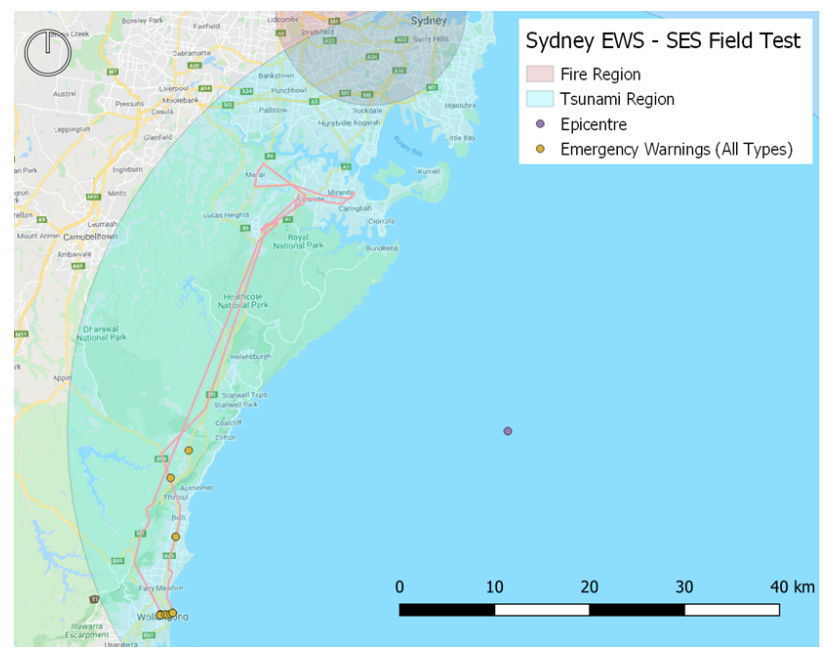

Figure 7. Route of the SES personnel during Sydney EWS test

Figure 8 displays screenshots from the app, showing successful reception, decoding, and display of the EWS message contents, as both decoded text and a map-view with the warning overlayed as an ellipse.

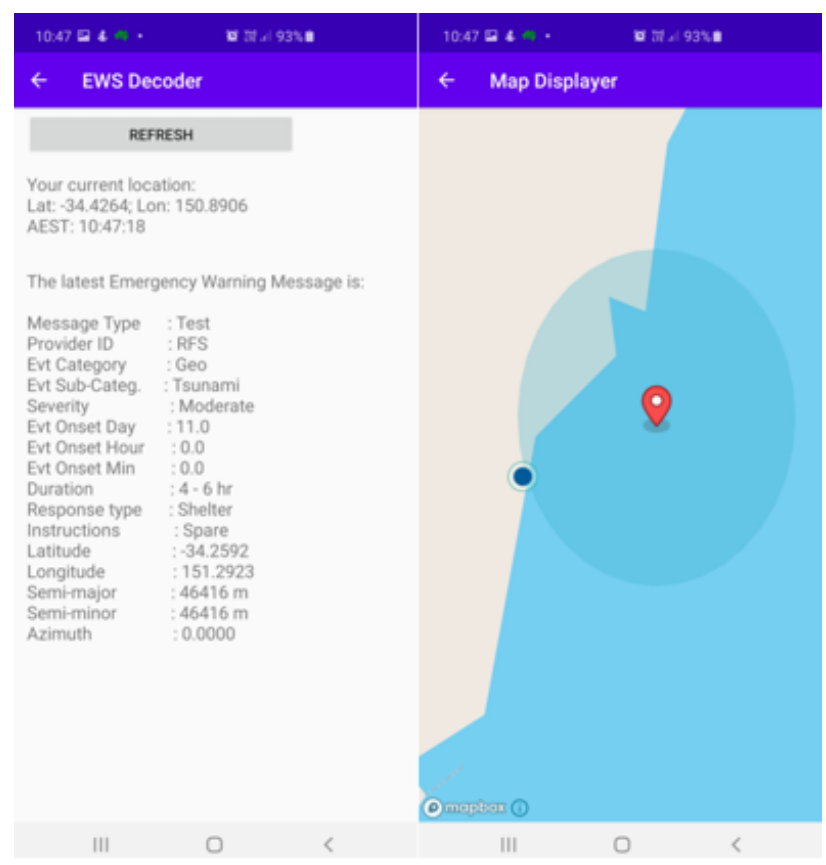

Figure 8. MobileApp screenshots during the Sydney EWS test

In general terms, the messages were received, but there were some technical difficulties experienced throughout the day, primarily related to the app. There was some confusion as to when to expect to receive messages, the expected type of message, and where the actual boundary of the event was located. Some of these challenges are elaborated upon in 
Section 5. The un-filtered logs from the device were received and studied by the research teams at RMIT and UNSW for further app development.

\section{CHALLENGES}

While the MobileApp worked mostly as expected at this early stage, the testers had identified several issues. These are summarised in this section.

(1) Event coverage not consistent with the methodology document provided.

Testers reported that the map-view displayed in the app was not consistent with the methodology document, and that the emergency warnings were received outside of the displayed emergency ellipse extents.

There were two main reasons for this. Firstly, different map projections were used in the methodology document, and the MobileApp. The methodology document used a GDA2020 / MGA Zone 56 (EPSG:7856) projection, while the app is using Web (or Pseudo) Mercator (EPSG:3857). In the first case, circles with a known radius are correctly displayed as a circle, but in the second, they become ellipses due to map distortion (see Figure 9 below). This issue can be fixed with further development of the MobileApp.

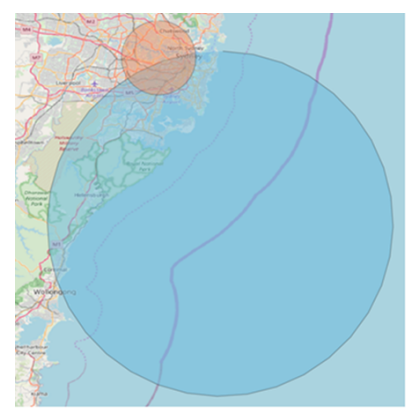

WSG84

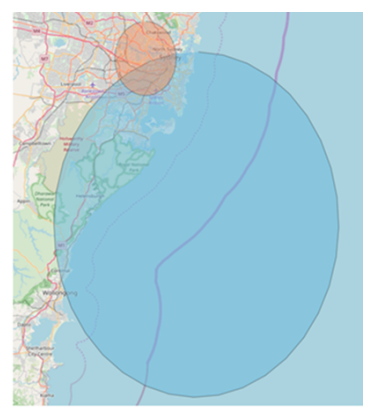

Pseudo Mercator
Figure 9. Differences between WGS84 and Pseudo Mercator.

The second reason is that the initial implementation of the checks on whether the user's (specifically the QZSS receiver's) location is within the coverage area, is not done using a strict polygon shape, but a bounding box. The main reason for this is to avoid memory intensive computations, but this approach also allows some buffer in which the messages are decoded if the user is nearby to the ellipse. These issues can be fixed with further development of the MobileApp.

(2) The Tsunami message continued to be displayed, despite the message not being actively sent.

The SES tester reported that the Tsunami message was shown during the scheduled slot for the bushfire, as shown in Figure 10. The messages alternated during the test day, with the bushfire alerts scheduled to be displayed between $3 \mathrm{pm}$ and $4 \mathrm{pm}$.

This is also an expected performance, as the app was designed to read the recorded QZSS message logs and to display the latest event detected nearby to the user's position. This was the chosen approach for the alpha version of the test app, as it helped mitigate hardware disconnection issues between the QZSS/GNSS receiver and the phone. As a result, as long as there was no new local warning message detected, the previously received message is the one that was displayed in the app. This can be easily managed by including a "Time of Reception" field in the decoded message view.

This was expected, given the limited resource and testing time and while there was no clear explanation for this behaviour it is considered solvable by further MobileApp development.

(3) Interaction between receiver reception and phone networks (Wi-Fi, Bluetooth, 4G).

One tester reported that the hardware connection might be unreliable when using multiple network connections, such as Wi-Fi or Bluetooth. However their testing results showed any effect to be intermittent or unrelated to the state of these connectivity settings. This behaviour will also need to be investigated during further MobileApp development.

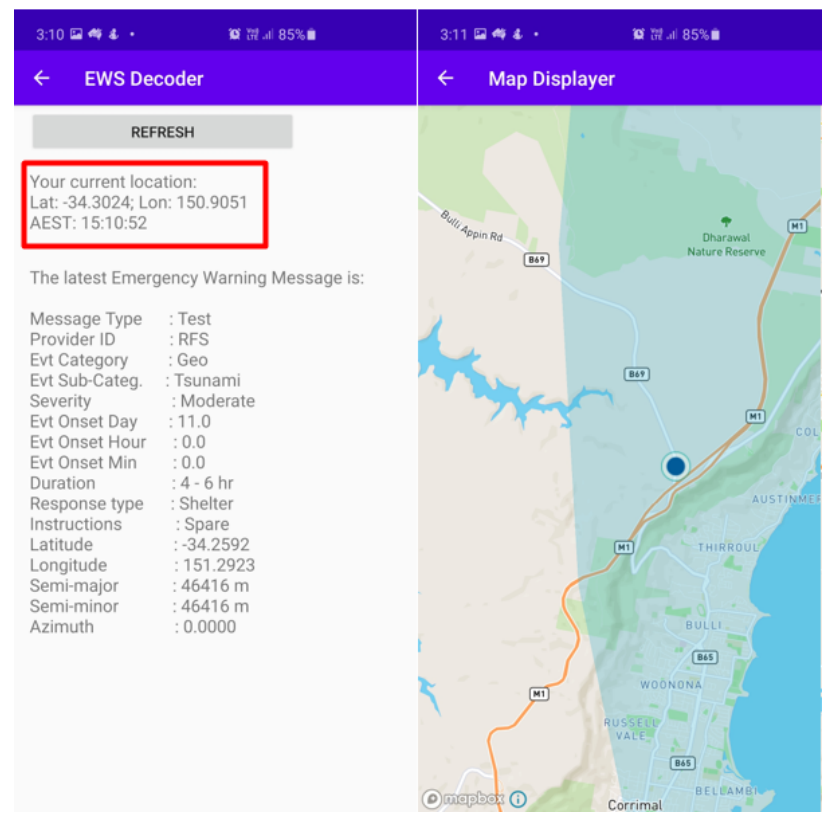

Figure 10. Tsunami Message decoded and displayed during Fire Message timeslot (3pm-4pm)

(4) The USB cable connection to a mobile phone is difficult to use in the field.

Many of the reliability issues experience by testers were inherent to the Softbank receivers used in the trial. This is consistent with behaviours observed in the early bench-tests, and could not be fully mitigated by a correct software implementation in the app. Other compatible receivers can be used that will allow use of a Bluetooth connection, which is preferable as it does not have cables. This would need to be considered in further usability design and any limitations removed for the user. This issue is considered solvable in a subsequent phase.

\section{CONCLUSIONS}

The first part of the project was to identify and engage with the relevant and appropriate representatives of interested stakeholders who would benefit from the deployment of an EWS via satellites such as QZSS; and to assess the feasibility of the QZSS EWS technology for transmission of warning messages in Australia. This component of work also examined technical aspects on the workflow, which include an understanding of the common alert messaging standard, 
designing of the Australian tailored EWS messages for QZSS satellite transmission, to receiving and decoding of the warning messages using a suitable equipment and mobile software application.

A field test campaign was carried out with cooperation with the emergency management services personnel such as from the NSW RFS, EMA and others. The aims of the testing campaign were to first prove that the EWS messages can be delivered via satellite and received by suitable devices; and second, to understand how these messages could be used by the end-users.

This paper summarised the activities of the Australia-Japan 2020 QZSS EWS trial project completed as part of a larger national initiative to implement nation-wide satellite-based EWS in Australia. The objectives of the test campaigns were to conduct an assessment of the QZSS EWS technology and its associated hardware usage in Australia; build capacity and to test the reception of the EWS messages in the field by the emergency management personnel in order to obtain feedback on the practicality of use in the field.

Both phases were successfully completed and a summary of findings is as follow:

- Australian tailored EWS messages could be transmitted via QZSS satellites for broadcast in Australia;

- The QZSS EWS signals can be received in Australia using a QZSS EWS enabled device, such as the Softbank receivers;

- The emergency messages embedded in the EWS signal can be decoded with an suitably designed mobile application such as the developed Andriod MobileApp;

- The emergency message containing type of disaster, start of disaster, event location, area of effect and user location could be continuously visualised as a text message and displayed spatially on a map interface on a mobile device;

- The trial revealed that the emergency warning message could be received at any location in Australia. Thus additional logic could include in future development of the MobileApp to ensure that only users in the affected area would receive the warning message. In other words, the warning messages can be tailored to specific region and locality within a defined geographical area;

- The field test have also identified some technical issues with the MobileApp due to its alpha stage, which can rectified and improved in the next phase of the project. For example, it became evident that an app needs to be developed to interact with the hardware and have a meaningful means of using the receiver in the field.

\section{OPPORTUNITIES AND RECOMMENDATIONS}

Australia's vast landmass possesses a significant operational and practical challenge to provide an efficient emergency warning mechanism that can disseminate time critical warning information 'wherever to whomever it is necessary' during events of disaster. It would require significant investment in establishing the ground communication network and underlying infrastructure, which may not be economically viable. The provision of warning messages through a satellite-based channel such as the QZSS EWS is feasible as it overcomes some of the weaknesses of mobile telephone-based services while combining the strength of both ground and space-based services. The key advantage of satellite-based communication is its high resilience to communication network overload and failure of ground systems and network infrastructure during a disaster. It also provides scalability of coverage, i.e. regional to national scale, as well as for mass public warning and its operation is potentially more cost effective compared to other warning systems.

In spite of pervasive presence of GNSS based technologies in other industry sectors to support positioning and navigation, application of GNSS in emergency warning and alerting in Australia is still not yet mature. To a large extent, research into the viability and implications of GNSS technology within the national emergency warning apparatus is still limited and on going. This boils down to the partial immaturity of the GNSS EWS technology as well as the availability of the EWS enabled devices and receivers in the market. However the challenge remains in thinking beyond the immediate barriers, identifying technical and non-technical requirements and understanding operational context in which the technology can be used as 'added value' to existing warning systems. It is expected that this soon will change, with the advent of new GNSS satellites and augmentation systems such as the Australian and New Zealand Satellite Positioning Augmentation Network and the EU's Galileo system. The Galileo-based EWS would be global in its scope, resilient to ground damage and deployable in response to all types of hazards, from earthquakes to bushfires and terrorist attacks. It is planned that the Galileo EWS service would reach a fully operational service in the 2023 timeframe and both Japan's QZSS and EU's Galileo EWS will adopt a common alert messaging standard (GSA, 2018; Japan Cabinet Office, 2019).

One of the key advantages of GNSS EWS technology is that it has the capabilities of delivering warning messages to people's personal mobile devices, from global to regional and local scale. The devices could be tailored to receive location-based emergency warnings at a specific point in time and within a defined geographical area. It has the potential to therefore address some of the shortcomings of the traditional warning services. A GNSS-based warning system is not likely to replace existing systems, but it can augment and strengthen them by providing an independent means of sending location-based warnings. There is a constant need to build effective warning systems that evolve over time by embracing newer technologies such as satellite-based technology.

On this, the recommended next steps are:

(1) to further engage and consult with relevant stakeholders to identify potential pathways for GNSS EWS technology adoption and implementation in Australia;

(2) to further advance the development of the MobileApp, which should be fit for purpose based on lessons learnt from this project, as well as feedback from end-users;

(3) to investigate options for extending the MobileApp towards integration with ground-based information and providing specialised instructions with respect to the endusers being emergency responder and/or a citizen;

(4) to establish strategic and operational plans for GNSS EWS implementation in Australia; and

(5) to promote the feasibility of the GNSS EWS technology and encourage adoption. 


\section{ACKNOWLEDGEMENTS}

The following people are acknowledged for providing support throughout the project: Hsi Lim and John Dawson from Geoscience Australia; Tommi Sullivan and Masahito Saito from Softbank; Reiko Shimada from Austrade; Gareth Carter from NSW SSG; Melissa Daley from NSW SES; Natalie Hill from NSW RFS; Leighton Bush from EMA; and Graeme Kernich from FrontierSI.

\section{REFERENCES}

Buist, P., Kohtake, N., Jagtman, E., 2013. Integrating Navigation and Communication for Emergency Services. 64th International Astronautical Congress, Beijing, China, 23-27 September.

Choy, S., Handmer, J., Whittaker, J., Shinohara, Y., Hatori, T., Kohtake. N., 2016. Application of satellite navigation system for emergency warning and alerting. Computers Environment and Urban Systems 58: 12-18.

Domínguez, E., Simón, F.J., Thomas, P., Zheng, Y., Wittmann, E., Lekaim, D., Tossaint, M., Jeannot, M. 2013. ESA's MultiConstellation Regional System Land Users Test-Bed Integrity Algorithms Experimentation Results. 26th International Technical Meeting of The Satellite Division of the Institute of Navigation (ION GNSS), Nashville, Tennessee, 16-20 September.

GSA, 2018. GNSS Emergency Warning Service successfully tested. https://www.gsa.europa.eu/ newsroom/news/gnss-emergency-warning-service-successfullytested (October 2020).

Handmer, J., Choy, S., Kohtake, H., 2014. Updating warning systems for climate hazards: Can navigation satellites help?. Australian Journal of Telecommunications and the Digital Economy 2(4), 70.1-70.14.

Iwaizumi, D., Ishida, T., Iino, S., Kohtake, N., Buist, P., 2014. GNSS-based Emergency Message Service: Lesson Learned and Future Prospects, 7th Advanced Satellite Multimedia Systems Conference. 13th Signal Processing for Space Communications Workshop, Livorno, Italy, 8-10 September.

Iwaizumi, D., Kohtake, N., 2013. Evaluation of reachability and promptness in delivering disaster and evacuation information using an augmentation signal of the QZSS system. 64th International Astronautical Congress. Beijing, China, 23-27 September.

Japan Cabinet Office, 2020. Quasi-Zenith Satellite System Performance Standard (PS-QZSS) and Interface Specification (IS-QZSS). https://qzss.go.jp/en/technical/ ps-is-qzss/ps-is-qzss.html (July 2020)

Japan Cabinet Office, 2019. Status Update on the QZSS Emergency Warning Services. $14^{\text {th }}$ Meeting of the

International Committee on Global Navigation Satellite Systems (ICG), Working Group B: Enhancement of performance of GNSS services, Bangalore, India, 10-12 December. https://qzss.go.jp/en/technical/ps-is-qzss/ ps-is-qzss.html

(October 2020)
Mathur, A.R., Traveset-Ventura, J., Montefusco, C., Toran, F., Plag, H-P., Ruiz, L., Stojkovic, I., Levy, J.C., 2005. Provision of Emergency Communication Messages through SBAS: The ESA ALIVE Concept. $18^{\text {th }}$ International Technical Meeting of The Satellite Division of the Institute of Navigation (ION GNSS). Long Beach, California, 13-16 September.

Wallner, S., 2011. Status of Definition of Dissemination of Emergency Alerts through GNSS, $6^{\text {th }}$ Meeting of the International Committee on Global Navigation Satellite Systems (ICG), Working Group B: Enhancement of performance of GNSS services, Tokyo, Japan, 5-9 September. 